\title{
citSATdb: Genome-Wide Simple Sequence Repeat (SSR) Marker Database of Citrus Species for Germplasm Characterization and Crop Improvement
}

\author{
Naveen Duhan ${ }^{1,2}$, Manish Meshram ${ }^{3}$, Cristian D. Loaiza ${ }^{1,2}$ and Rakesh Kaundal 1,2,3,*(1) \\ 1 Department of Plants, Soils and Climate, Utah State University, Logan, UT 84322, USA; \\ naveen.duhan@aggiemail.usu.edu (N.D.); cdloaiza@aggiemail.usu.edu (C.D.L.) \\ 2 Center for Integrated BioSystems (CIB), Utah State University, Logan, UT 84322, USA \\ 3 Department of Computer Science, Utah State University, Logan, UT 84322, USA; \\ manish.meshram@aggiemail.usu.edu \\ * Correspondence: rkaundal@usu.edu; Tel.: +1-(435)-797-4117; Fax: +1-(435)-797-2766
}

Received: 13 November 2020; Accepted: 26 November 2020; Published: 10 December 2020

\begin{abstract}
Microsatellites or simple sequence repeats (SSRs) are popular co-dominant markers that play an important role in crop improvement. To enhance genomic resources in general horticulture, we identified SSRs in the genomes of eight citrus species and characterized their frequency and distribution in different genomic regions. Citrus is the world's most widely cultivated fruit crop. We have implemented a microsatellite database, citSATdb, having the highest number $(\sim 1,296,500)$ of putative SSR markers from the genus Citrus, represented by eight species. The database is based on a three-tier approach using MySQL, PHP, and Apache. The markers can be searched using multiple search parameters including chromosome/scaffold number(s), motif types, repeat nucleotides (1-6), SSR length, patterns of repeat motifs and chromosome/scaffold location. The cross-species transferability of selected markers can be checked using e-PCR. Further, the markers can be visualized using the Jbrowse feature. These markers can be used for distinctness, uniformity, and stability (DUS) tests of variety identification, marker-assisted selection (MAS), gene discovery, QTL mapping, and germplasm characterization. citSATdb represents a comprehensive source of markers for developing/implementing new approaches for molecular breeding, required to enhance Citrus productivity. The potential polymorphic SSR markers identified by cross-species transferability could be used for genetic diversity and population distinction in other species.
\end{abstract}

Keywords: SSR; citrus; marker-assisted selection; database; microsatellites; genomics; plant breeding and genetics

\section{Introduction}

The genetic selection of plants in conventional plant breeding is decided by the parents and influenced by different environmental conditions [1]. In conventional plant breeding, alleles are mixed over the generations, resulting in the development of new combinations, which helps in achieving higher trait value through selection. Developing a variety of woody plant species through traditional breeding may take 1012 years [2]. This time period can be reduced by performing marker-assisted selection (MAS) on seedling material [3]. In recent years, marker-assisted selection has become popular in breeding programs for many crops [4-7]. One of the pre-requisites for using MAS is the discovery of DNA-based markers, which are tightly linked to the target trait of interest. Microsatellite (SSR) markers have been the system of choice for quantitative trait loci (QTL) mapping in many crops for a 
long time. Microsatellites are tandem repeats of 1-6 nucleotide long DNA units flanked by unique sequences in the genome but found more abundantly in the intronic region [8]. These are characterized by multi-allelic variation, reproducibility, and a high co-dominant inheritance [9]. The mutation rate of SSRs ranges from $10^{-3}$ to $10^{-6}$ per generation [10], which increases with the length of the repeat unit [11]. They are highly versatile, low-cost, informative PCR-based molecular markers associated with a high frequency of length polymorphism [12]. These features make them the preferred choice among available genetic markers (e.g., AFLP, RAPD, RFLP, SNP and SRAP) and provide the basis for their effective applications in a wide range of fields, such as genetic mapping, QTLs identification, varietal identification, genetic diversity analysis, linkage mapping, marker-assisted selection and evolutionary analysis [13].

The genus Citrus is a large taxonomic rank that includes many cultivated species such as oranges, lemons, pomelos, grapefruits, and limes. Citrus plants are woody, perennial small to moderate-sized trees that are cultivated all over the world to produce fresh fruits and juice, and as ornamentals, etc. Being part of our heritage, the citrus industry has a great social and cultural significance, in addition to its economic and agronomic importance. A number of SSR markers have been reported in citrus in different studies, but there is a limited catalogue. Therefore, there is a need for more comprehensive identification of markers for genetic diversity, MAS, association or comparative mapping, genetic linkage maps, and qualitative and quantitative traits [14-22].

Conventional methods for SSR screening using genomic libraries are costly, labor-intensive and time-consuming [23]. In silico approaches can thus be used to overcome this problem. These methods have the advantage of predicting SSRs in specific regions in the genome, which is more efficient in designing molecular markers for linkage mapping and QTLs [24]. The advent of next-generation sequencing (NGS) technologies and advancement in computational approaches have made possible the discovery of markers in bulk.

In accordance with the TRIPS (trade-related aspects of intellectual property rights) agreement and other intellectual property rights, plant breeders own a variety on the basis of distinctness, uniformity and stability (DUS) characteristics, which can be used for allocating new variety status and solving legal disputes [25]. So, in order to supplement DUS characteristics for variety identification, SSR markers were successfully used in crops such as rice [26], maize [27], barley [28], tobacco [29], soybean [30], wheat [31], mung beans [32], kadam [33] and potatoes [34]. The characterization of different Citrus species can also be undertaken using such approaches. The Citrus genus contains more than 100 species with limited genomic resources available. Extensive molecular mining of SSR markers and assessment of their polymorphism with cross-species transferability may be a more pragmatic approach to addressing the need for markers in previously untouched species. In closely related species, flanking regions of SSRs are conserved; heterologous primers of these flanking regions can facilitate the use of molecular markers [35]. SSR markers from focal species can be applied in non-focal species to investigate the population genetic structure of wild species [36].

Though different microsatellite databases have been developed [37,38], due to the high number of species in these databases, they lack some features, such as e-PCR, browsing/visualizing SSRs on the genome, etc. Therefore, in addition to the existing databases, a dedicated database focusing on all available genomic data of the genus Citrus that contains a wide range of user-friendly features could be a valuable genomic resource for Citrus crop improvement and characterization.

The present study was aimed at genome-wide mining of SSRs and the development of a user-friendly database containing microsatellites from eight Citrus species (Citrus sinensis, Citrus clementina, Citrus maxima, Citrus medica, Citrus ichangensis, Atlantia buxifolia, Citrus reticulata and Fortunella hindsii) with the options for chromosome-wise SSR mining and primer designing for genotyping, along with e-PCR-based polymorphism discovery. It also aims to provide annotated genic regions of SSR to be used as functional domain markers (FDMs). 


\section{Materials and Methods}

\subsection{Data Collection}

The genomes of Citrus sinensis [39], Citrus clementina [40], Citrus maxima, Citrus medica, Citrus ichangensis, and Atlantia buxifolia [41] were downloaded from the Citrus Genome Database [42]; Citrus sinensis and Citrus maxima were assembled chromosome-wise while Citrus medica, Citrus clementina, Citrus ichangensis, and Atalantia buxifolia were assembled scaffold-wise, Citrus reticulata through pseudomolecule assembly and Fortunella hindsii through contig-assembly (Table 1). Genome assemblies were used from these two resources: Huazhong Agricultural University (HZAU), and Joint Genome Institute (JGI).

Table 1. The genomic data of different Citrus species used in the study.

\begin{tabular}{ccccc}
\hline Species & Assembly Version & Assembly Level & Genome Size (Mb) & GC\% \\
\hline Citrus sinensis & v2.0 (HZAU) & Chromosome & 327.945 & $34.06 \%$ \\
\hline Citrus clementina & v1.0 (JGI) & Scaffold & 301.387 & $34.96 \%$ \\
\hline Citrus maxima & v1.0 (HZAU) & Chromosome & 345.78 & $34.99 \%$ \\
\hline Citrus medica & v1.1 (HZAU) & Scaffold & 406.058 & $35.16 \%$ \\
\hline Citrus ichangensis & v1.0 (HZAU) & Scaffold & 357.621 & $34.21 \%$ \\
\hline Atalantia buxifolia & v1.1 (HZAU) & Scaffold & 315.821 & $33.55 \%$ \\
\hline Citrus reticulata & v1.1(HZAU) & Pseudomolecule & 334.2 & - \\
\hline Fortunella hindsii & V1.1(HZAU) & Contig & 373.6 & $34.49 \%$ \\
\hline
\end{tabular}

\subsection{In Silico Simple Sequence Repeat Mining and Primer Designing}

SSRs were identified in genomes of 8 citrus species (Table 1). A Perl script (miSATminer) was written to identify repeat motifs in a genome sequence. Microsatellites were identified with parameters such as 10 repeat units for mono, 5 repeat units for the di, tri, tetra, penta, and hexa. In-house Perl scripts were used to fetch the flanking regions of the identified SSRs for primer designing. Primer3 executables were used to design primers with the following default parameters: melting temperature, 55-65 ${ }^{\circ} \mathrm{C}$; GC content, 40-60\%; primer size, 18-27 bp; length and product size, 150-280 bp [43].

\subsection{Functional Annotation of SSR Markers}

The full annotation of gene functions is available for these eight citrus species and was implemented in the Jbrowse genome browser inside the citSATdb database. In this genome browser, markers can be visualized against the reference sequence, gene coordinates, and structural and functional details.

\subsection{Marker and Database Development Workflow}

Microsatellite repeat loci were mined by pattern identification in the genome sequences using miSATminer, our inhouse developed Perl script. This script mines SSR loci from the genome sequences with custom repeat parameters. SSR primers for genotyping were designed using Primer3 executables by extracting a flanking length of $300 \mathrm{bp}$ upstream and $300 \mathrm{bp}$ downstream of SSR loci in the genome. Selected repeats can be viewed with their markers in the sequence. ePCR was implemented in the database for polymorphism. In citSATdb, we have provided eight genome assemblies and an option for uploading user sequences to check amplification. The markers with variable product size in two genomes were considered as polymorphic markers. All the results can be downloaded as a CSV file. The whole workflow of the database is depicted in (Figure 1). 


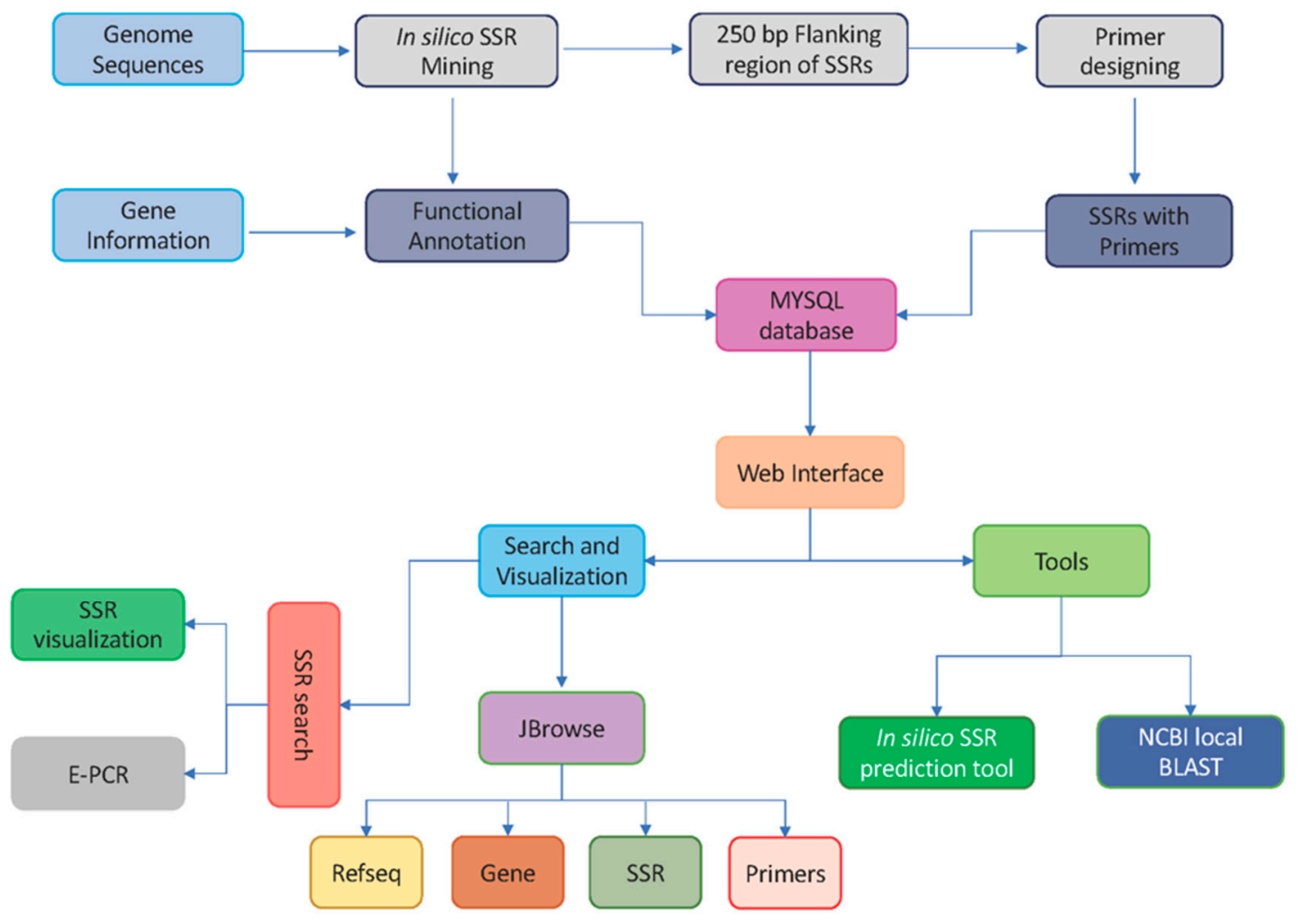

Figure 1. The schematic workflow of $c i t S A T d b$ (citrus microSATellite) database.

\subsection{Database Development and Web Interface}

The Citrus microsatellite database ( $\operatorname{citSATdb}$ ) is a three-tier-based relational database developed with a client tier, middle tier, and database tier. Predicted SSRs and their corresponding primers were stored in MySQL data tables and accessed through the Apache server. A user-friendly interface of the database was developed with PHP, HTML5, and Jquery. In silico microsatellite designing with miSATminer and custom Perl scripts and Primer3 was implemented for primer designing. Jbrowse for the visualization of genomic sequences, SSRs and primers was also implemented. The NCBI local and remote database was also implemented for similarity searches. e-PCR was implemented for cross-species transferability. The web server contains seven tabs viz. Home, About, Species, Tools, JBrowse, Help, and Contact; the database will be updated regularly with newly available genome data.

\section{Results and Discussion}

\subsection{Cross-Species Comparison of Citrus Species SSRS}

For the development of the Citrus web genomics resource, SSR loci were mined successfully using miSATminer. A total of 1,699,853 putative microsatellites were mined from the genomes of eight Citrus species. The highest number of microsatellites were identified in Fortunella hindsii $(240,182)$, followed by Citrus ichangensis (226,950), Citrus maxima (224,961), Citrus medica (210,590), Atalantia buxifolia $(204,687)$, Citrus sinensis $(203,297)$, Citrus reticulata $(201,408)$ and Citrus clementina $(187,778)$. Maximum microsatellite density (SSRs/Mb) was observed in Atalantia buxifolia (675.26), whereas the minimum was observed in Citrus medica (552.12) (Table 2). Previous studies have reported a negative correlation between the SSR density and genome size [44]. However, the SSRs identified in our study of eight Citrus species show no correlation between the SSR density and genome size. This is in line with some of the recent findings which reported that there is no correlation between the genome size and SSRs density; genome size differences may lead to the degree of microsatellite repetition in the genome [45-50]. 
Table 2. Distribution of identified SSRs in Citrus species.

\begin{tabular}{ccccc}
\hline Species & Predicted SSRs & Designed Markers & SSRs/MB & Genome Size (MB) \\
\hline Citrus sinensis & 203,297 & 157,003 & 619.91 & 380 \\
\hline Citrus clementina & 187,778 & 150,593 & 638.15 & 370 \\
\hline Citrus maxima & 224,961 & 184,840 & 650.59 & 328 \\
\hline Citrus medica & 210,590 & 143,826 & 552.12 & 380 \\
\hline Citrus ichangensis & 226,950 & 157,810 & 648.06 & 407 \\
\hline Atalantia buxifolia & 204,687 & 147,140 & 675.26 & 391 \\
\hline Citrus reticulata & 201,408 & 158,143 & 602.66 & 370 \\
\hline Fortunella hindsii & 240,182 & 197,145 & 642.89 & 370 \\
\hline
\end{tabular}

\subsection{SSR Motifs Characterized by Repeat Length}

In all the species, mono-nucleotide repeats were most abundant, followed by di-, tri-, tetra-, penta-, and hexa-nucleotide repeats. Among all the citrus species, the maximum number of mono-nucleotide repeats was found in Fortunella hindsii $(152,611)$ followed by C. ichangensis $(144,115)$, C. maxima $(142,446)$, A. buxifolia $(129,304)$, C. medica $(125,467)$, C. reticulata $(123,469)$, C. sinensis $(121,051)$, and C. clementina $(115,888)$. In the case of di-nucleotide repeats, the maximum number was observed in F. hindsii $(61,408)$ followed by C. medica $(60,040)$, C. ichangensis $(57,930)$, C. maxima $(57,059)$, C. reticulata $(55,336), C$. sinensis $(54,874), A$. buxifolia $(51,030)$, and C. clementina $(50,108)$. The occurrence of tri-nucleotides was observed highest in C. sinensis $(23,568)$ followed by $F$. hindsii $(22,143), C$. maxima $(21,668), C$. medica $(21,259)$, C. ichangensis $(21,149)$, A. buxifolia $(20,572)$, C. reticulata $(19,400)$, and C. clementina $(18,553)$. Similarly, tetra-nucleotides were most frequent in F. hindsii (3159) followed by C. sinensis (3050), C. medica (2975), C. maxima (2954), C. ichangensis (2844), A. buxifolia (2822), C. reticulata (2631), and C. clementina (2620). C. ichangensis (613) and A. buxifolia (613) contain the maximum penta-nucleotides, followed by F. hindsii (576), C. maxima (539), C. medica (534), C. sinensis (473), C. clementina (436), and C. reticulata (408). Hexa-nucleotides were most abundant in A. buxifolia (346) followed by C. medica (315), C. ichangensis (299), C. maxima (295), F. hindsii (285), C. sinensis (281), C. clementina (173), and C. reticulata (164) (Table 3, Figure 2). From these results, a high abundance of mono-nucleotide repeats was observed in all the genomes, which may be due to the intrinsic limitation of the chemistry of next-generation sequencing (NGS) technology used for data generation [51]. Similarly, di-nucleotide repeats in higher abundance have also been reported in other crops [52,53].

Table 3. Distribution of predicted SSR motif types in each of the Citrus species.

\begin{tabular}{ccccccc}
\hline Species & Mono & Di & Tri & Tetra & Penta & Hexa \\
\hline Citrus sinensis & 121,051 & 54,874 & 23,568 & 3050 & 473 & 281 \\
\hline Citrus clementina & 115,888 & 50,108 & 18,553 & 2620 & 436 & 173 \\
\hline Citrus maxima & 142,446 & 57,059 & 21,668 & 2954 & 539 & 295 \\
\hline Citrus medica & 125,467 & 60,040 & 21,259 & 2975 & 534 & 315 \\
\hline Citrus ichangensis & 144,115 & 57,930 & 21,149 & 2844 & 613 & 299 \\
\hline Atalantia buxifolia & 129,304 & 51,030 & 20,572 & 2822 & 613 & 346 \\
\hline Citrus reticulata & 123,469 & 55,336 & 19,400 & 2631 & 408 & 164 \\
\hline Fortunella hindsii & 152,611 & 61,408 & 22,143 & 3159 & 576 & 285 \\
\hline
\end{tabular}



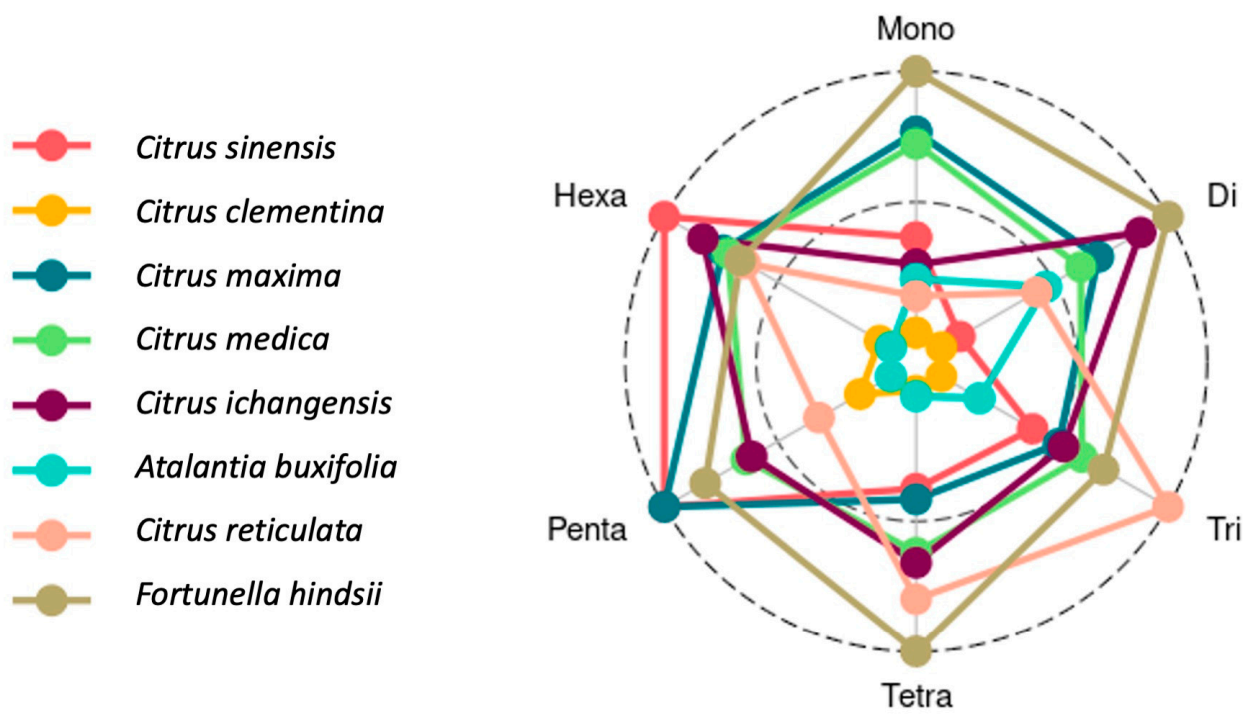

Figure 2. Distribution of SSR motifs in each of the Citrus species.

\subsection{Designed SSR Primers, Motif Characterization by Repeat Length}

$c i t S A T d b$ is a comprehensive microsatellite database of Citrus represented by eight species containing 1,296,500 in silico predicted markers. Distribution-wise, mononucleotide repeat primers were the most abundant followed by di-, tri-, tetra-, penta-, and hexa-nucleotide. Among the eight species, the maximum number of mononucleotide repeat primers were designed in F. hindsii $(128,597)$ followed by C. maxima (120,885), C. ichangensis $(100,422)$, C. reticulata $(98,016)$, C. sinensis $(97,149), C$. clementina $(96,191)$, A. buxifolia $(95,905)$, and C. medica $(85,972)$. In the case of di-nucleotide primers, the maximum number was observed in F. hindsii $(50,791)$ followed by C. maxima $(45,399), C$. reticulata $(44,528)$, C. sinensis $(44,198), C$. medica $(40,922)$, C. ichangensis $(40,333)$, C. clementina $(38,870)$, and $A$. buxifolia $(37,363)$. The occurrence of tri-nucleotides motif primer was observed highest in C. maxima $(16,037)$ followed by F. hindsii $(14,639)$, C. ichangensis $(14,404)$, C. medica $(14,248)$, C. clementina $(13,418), C$. reticulata $(13,171), C$. sinensis $(13,069)$, and A. buxifolia $(11,120)$. Similarly, tetra-nucleotides primers were most frequent in F. hindsii (2390) followed by C. medica (2097), A. buxifolia (2050), C. sinensis (2022), C. ichangensis (2002), C. reticulata (1981), C. maxima (1979), and C. clementina (1722). F. hindsii (494) contains maximum penta-nucleotides primers followed by A. buxifolia (446), C. ichangensis (426), C. sinensis (385), C. medica (363), C. maxima (352), C. reticulata (310), and C. clementina (285). Hexa-nucleotides primers were most abundant in A. buxifolia (256) followed by F. hindsii (234), C. medica (224), C. ichangensis (223), C. maxima (188), C. sinensis (180), C. reticulata (137), and C. clementina (107) (Table 4, Figure 3).

Table 4. Distribution of motif types in the designed SSR markers for each of the Citrus species.

\begin{tabular}{ccccccc}
\hline Species & Mono & Di & Tri & Tetra & Penta & Hexa \\
\hline Citrus sinensis & 97,149 & 44,198 & 13,069 & 2022 & 385 & 180 \\
\hline Citrus clementina & 96,191 & 38,870 & 13,418 & 1722 & 285 & 107 \\
\hline Citrus maxima & 120,885 & 45,399 & 16,037 & 1979 & 352 & 188 \\
\hline Citrus medica & 85,972 & 40,922 & 14,248 & 2097 & 363 & 224 \\
\hline Citrus ichangensis & 100,422 & 40,333 & 14,404 & 2002 & 426 & 223 \\
\hline Atalantia buxifolia & 95,905 & 37,363 & 11,120 & 2050 & 446 & 256 \\
\hline Citrus reticulata & 98,016 & 44,528 & 13,171 & 1981 & 310 & 137 \\
\hline Fortunella hindsii & 128,597 & 50,791 & 14,639 & 2390 & 494 & 234 \\
\hline
\end{tabular}




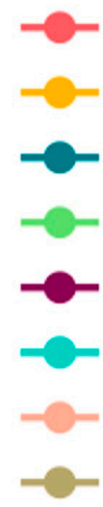

Citrus sinensis

Citrus clementina

Citrus maxima

Citrus medica

Citrus ichangensis

Atalantia buxifolia

Citrus reticulata

Fortunella hindsii

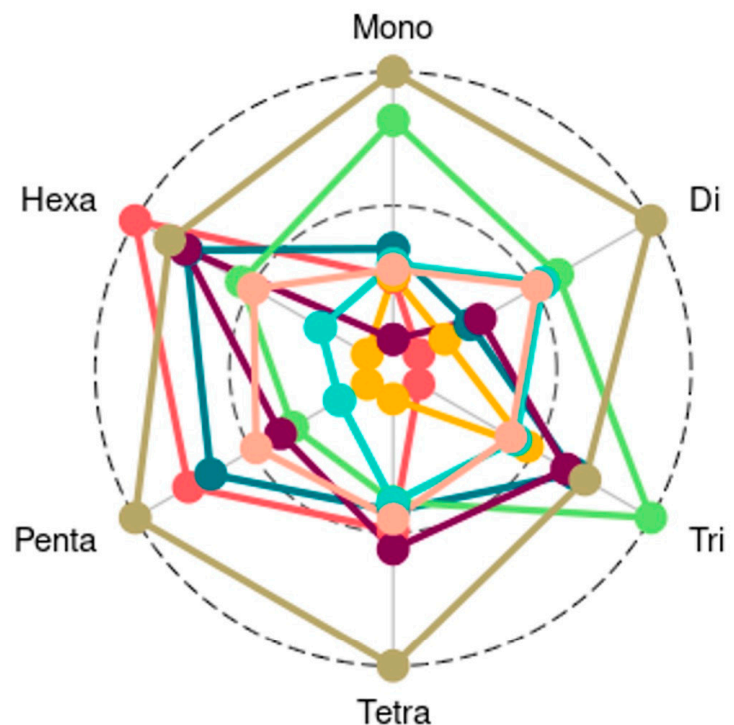

Tetra

Figure 3. Distribution of motif types in the predicted SSR markers.

The designed SSR primers can be used for QTL/candidate gene identification, linkage mapping, and germplasm characterization. Varieties with similar morphological characteristics are very difficult to differentiate from just the phenotypic study. To conquer these difficulties, SSR markers have been used in previous studies for variety characterization, trait improvement, linkage mapping, molecular breeding application, variety development, and phylogenetic and taxonomic comparisons [8,53-55]. Similarly, 24 SSR markers were used to assess genetic diversity in 370 Citrus accessions [19]. The designed putative primers present in $c i t S A T d b$ can be used in rapid genotyping for genetic diversity and differentiating varieties. Varietal differentiation using SSR markers has already been reported in many other crops, such as barley [56], sugarcane [57], eggplant [58], capsicum [59], and sesame [60]. These markers can be further explored for trait improvement averse to abiotic and biotic stresses. For example, in Satsuma mandarins, SSR has been used to discover one major QTL for male sterility, and such a QTL can be used in seedless citrus breeding by using flanking region SSR markers with allele size differences between donor and recipient varieties [61]. Such markers can be used for high-density linkage mapping and the discovery of genes needed to improve specific traits. Using SSRs, a linkage map was developed, and QTL mapping was performed to find loci related to the freezing tolerance of citrus [62].

The availability of whole-genome assemblies of different plant species in the public domain provides an opportunity for the study of cross-species transferability in closely related species. Trait-specific candidate genes may be cloned from different species [63]. In silico cross-species transferability can also be predicted with $c i t S A T d b$, which can be further used for phylogenetic and diversity studies. A similar use has been reported for diversity analysis in citrus species with few numbers of markers [19].

\subsection{Functional Annotation of SSRs and Markers}

All the predicted SSRs were mapped on the gene feature file (GFF) of each genome. In C. sinensis, 62,563 SSRs were found to be mapped onto the genic regions, followed by C. clementina $(45,975), C$. maxima $(60,654)$, C. medica $(49,403)$, C. ichangensis $(52,336)$, A. buxifolia $(53,937)$, C. reticulata $(53,832)$ and F. hindsii $(73,901)$ (Table 5). Further, we designed primers for each of the species; in C. sinensis $(48,804)$, C. clementina (42,476), C. maxima $(60,267)$, C. medica $(50,690)$, C. ichangensis $(54,065)$, A. buxifolia $(46,373)$, C. reticulata $(48,203)$, and F. hindsii $(67,288)$, genic SSR primers were designed. A detailed distribution of the predicted and designed markers on both the genic and non-genic regions is presented in (Table 5). 
Table 5. Distribution of SSRs in the individual Citrus genome.

\begin{tabular}{ccccc}
\hline \multirow{2}{*}{ Species } & \multicolumn{2}{c}{ Predicted Markers } & \multicolumn{2}{c}{ Designed Markers } \\
\cline { 2 - 5 } & Genic & Non-Genic & Genic & Non-Genic \\
\hline Citrus sinensis & 62,563 & 140,734 & 48,804 & 108,199 \\
\hline Citrus clementina & 45,975 & 141,803 & 42,476 & 108,117 \\
\hline Citrus maxima & 60,654 & 164,307 & 60,267 & 124,573 \\
\hline Citrus medica & 49,403 & 161,187 & 50,690 & 93,136 \\
\hline Citrus ichangensis & 52,336 & 174,314 & 54,065 & 103,745 \\
\hline Atalantia buxifolia & 53,937 & 150,750 & 46,373 & 100,767 \\
\hline Citrus reticulata & 53,832 & 147,576 & 48,203 & 109,940 \\
\hline Fortunella hindsii & 73,901 & 166,281 & 67,288 & 129,857 \\
\hline
\end{tabular}

\subsection{Comparison with Another Databases}

Many databases of marker development in plants are publicly available. The Pan-Species Microsatellite Database (PSMD) database contains eight Citrus species in its repository, although it lacks some features such as e-PCR, JBrowse, and BLAST. Plant micro-satellite Database (PMDbase) is another online database, but it has some limitations such as the markers search by user choice, repeat kind, motif type, location in the genome, etc. Secondly, only two species of citrus are present in this database. Similarly, SSRome also has only two Citrus species and lacks features such as ePCR, JBrowse, BLAST, etc. The citSATdb resource overcomes these limitations and is specifically designed as a user-friendly interface to assist the researchers in the horticultural sciences. A detailed comparison of PMDBase, PSMD, SSRome, and citSATdb is presented in (Table 6).

Table 6. Comparison of $c i t S A T d b$ with PMDBase, PSMD, and SSRome features.

\begin{tabular}{ccccc}
\hline Features & citSATdb & PMDBase & PSMD & SSRome \\
\hline Citrus species & 8 & 2 & 8 & 2 \\
\hline Search option with ID & $\sqrt{ }$ & $\times$ & $\sqrt{ }$ & $\times$ \\
\hline Search by motif type & $\sqrt{ }$ & $\times$ & $\sqrt{ }$ & $\sqrt{ }$ \\
\hline Search by repeat type & $\sqrt{ }$ & $\times$ & $\sqrt{ }$ & $\sqrt{ }$ \\
\hline Genic and genomic search & $\sqrt{ }$ & $\times$ & $\sqrt{ }$ & $\sqrt{ }$ \\
\hline Search on user-defined location & $\sqrt{ }$ & $\times$ & $\sqrt{ }$ & $\times$ \\
\hline Length of SSR & $\sqrt{ }$ & $\times$ & $\sqrt{ }$ & $\times$ \\
\hline ePCR option & $\sqrt{ }$ & $\times$ & $\times$ & $\times$ \\
\hline Non-nuclear (mitochondrion and chloroplast) & $\times$ & $\sqrt{ }$ & $\times$ & $\times$ \\
\hline JBrowse visualization of SSRs & $\sqrt{ }$ & $\times$ & $\times$ & $\times$ \\
\hline BLAST & $\sqrt{ }$ & $\times$ & $\times$ & $\times$ \\
\hline
\end{tabular}

\section{6. citSATdb: Citrus Microsatellite Web-Genomic Resource}

The citrus web-genomic resource (citSATdb) was developed successfully using a three-tier architecture. This is a comprehensive microsatellite database of Citrus represented by eight species containing 1,296,500 in silico predicted markers. The web server contains seven tabs viz. Home, About, Species, Tools, JBrowse, Help, and Contact. The 'Species' tab provides information about the selected species on left and search options on the right side. In silico predicted markers can be searched by selecting genic or genomic, chromosome/scaffold-wise, along with motif type, repeat type, length, and location in the genome. The search results provide a visualization of repeat and flanking primers on 
the sequence extracted with $500 \mathrm{bp}$ upstream and downstream of the repeat. It also provides an option for ePCR whereby users can check the in silico amplification of selected primers in the genome or cross-species transferability with the user-given sequence(s). All the results can be downloaded in a CSV format text file. The 'Tool' page provides two tabs-SSR prediction and BLAST. miSATminer was implemented with custom scripts to design SSRs and their primers for user input sequences. Standalone BLAST was implemented on the BLAST search page, where users can align their SSR query sequences to genomes. All the eight genome sequences can be visualized with gene and SSR coordinates on the genome using the 'JBrowse' table. The 'Help' tab contains a detailed tutorial for using the database efficiently and a list of frequently asked questions. A detailed workflow of exploring the $c i t S A T d b$ and its search features is illustrated in Figure 4.
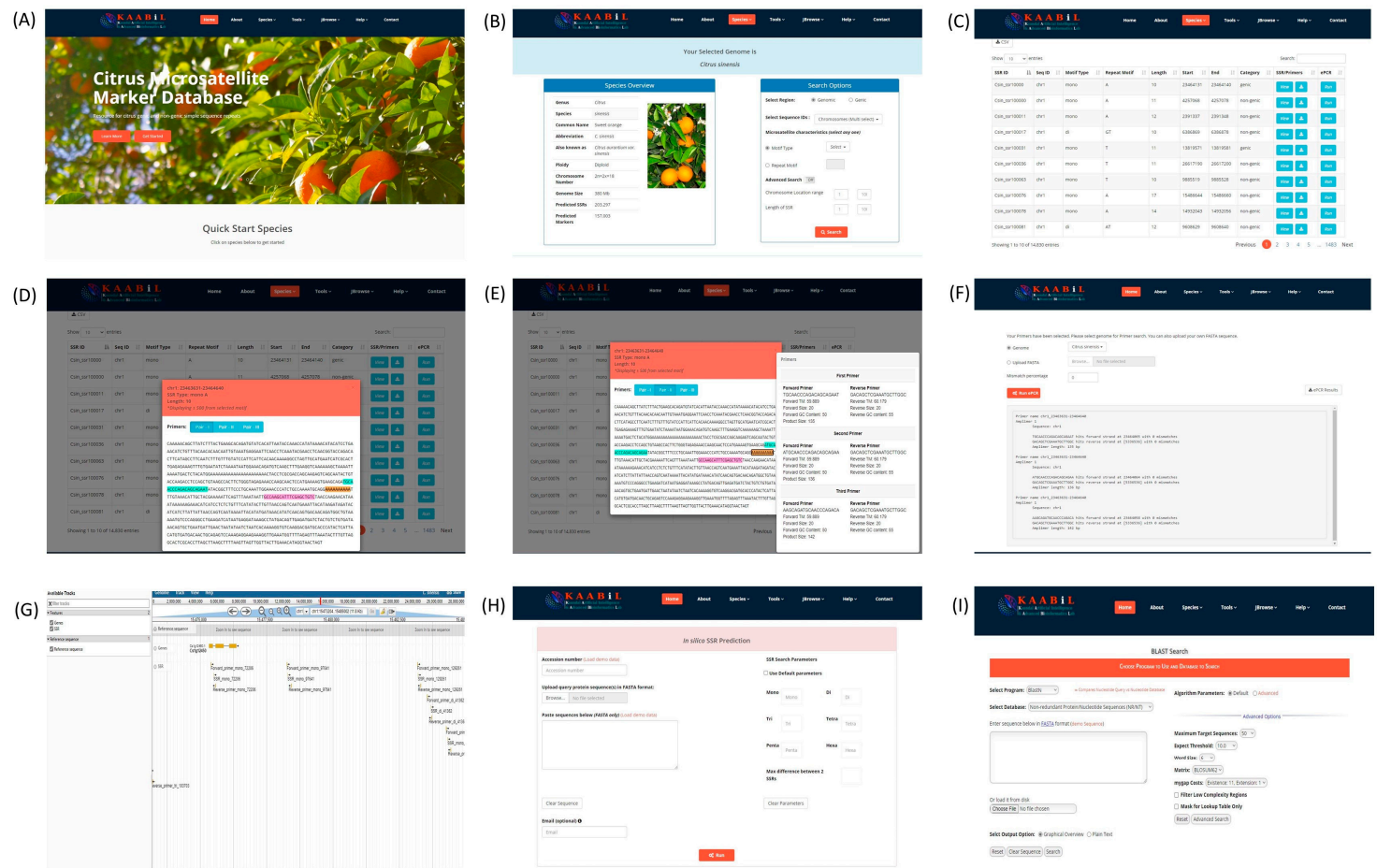

Figure 4. The main functions of the $c i t S A T d b$ database and search modules. (A) Home page; (B) species search page; (C) results page showing the desired search results; (D) results page displaying SSR and corresponding primers in sequence; $(\mathbf{E})$ sequence of primers and their features; $(\mathbf{F})$ ePCR results page; (G) JBrowse for visualization of markers on genome; $(\mathbf{H})$ miSATminer tool for SSR prediction; and (I) BLAST search page.

\section{Conclusions}

We report here a comprehensive web genomic resource for the genus Citrus covering three of its commercially important species. citSATdb, accessed freely via the address http://bioinfo.usu.edu/ citSATdb/, contains a total of 1,296,500 putative microsatellite DNA markers. Our findings on the cross-species transferability of microsatellite loci among six different species of Citrus can be used to cater to the need for molecular markers, especially for the more than 100 species of the genus Citrus for which there are no whole-genome sequence data available yet. This genomic resource can be of immense use to the global community. It can be used for chromosome-wise microsatellite locus mining and primer designing for non-genic and genic FDM-SSR for rapid genotyping. It can also be used to accelerate polymorphism discovery by e-PCR, thus being economically beneficial and needed in future re-sequencing projects. The database can be used not only for knowledge discovery research, such as QTL and gene mapping, but also for marker-assisted breeding in Citrus germplasm improvement and management. 
Author Contributions: N.D. and R.K. formulated and designed the research. N.D. and C.D.L. analyzed the data. N.D. and M.M. designed and constructed the web database. Writing-original draft preparation, N.D.; writing-review and editing, R.K.; visualization, N.D. and R.K.; supervision, R.K.; project administration, R.K.; funding acquisition, R.K. All authors have read and agreed to the published version of the manuscript.

Funding: The authors acknowledge the support to this study from the faculty start-up funds to RK from the Center for Integrated BioSystems (CIB)/Department of Plants, Soils, and Climate, USU. This research was also supported by the Utah Agricultural Experiment Station (UAES), and approved as journal paper number 9410. The funding body did not play any role in the design of this study, the collection, analysis, and interpretation of data, or in the writing of this manuscript.

Acknowledgments: The authors thank the anonymous referees for suggestions and help in improving the research article.

Conflicts of Interest: The authors declare no conflict of interest. The funders had no role in the design of the study; in the collection, analyses, or interpretation of data; in the writing of the manuscript, or in the decision to publish the results.

\section{References}

1. Collard, B.C.Y.; Septiningsih, E.M.; Das, S.R.; Carandang, J.J.; Pamplona, A.M.; Sanchez, D.L.; Kato, Y.; Ye, G.; Reddy, J.N.; Singh, U.S.; et al. Developing new flood-tolerant varieties at the international rice research institute (IRRI). Sabrao J. Breed. Genet. 2013, 45, 42-56.

2. Xiu, X.D. Advances in worldwide citrus breeding. Acta Hortic. Sin. 2005, 32, 1140-1146.

3. Johnson, R. Marker-Assisted Selection. In Plant Breeding Reviews; John Wiley \& Sons Inc.: Oxford, UK, 2010; pp. 293-309.

4. Cuenca, J.; Aleza, P.; Garcia-Lor, A.; Ollitrault, P.; Navarro, L. Fine Mapping for Identification of Citrus Alternaria Brown Spot Candidate Resistance Genes and Development of New SNP Markers for Marker-Assisted Selection. Front. Plant Sci. 2016, 7, 1948. [CrossRef] [PubMed]

5. Gupta, H.; Agrawal, P.K.; Mahajan, V.; Bisht, G.S.; Kumar, A.; Verma, P.; Srivastava, A.; Saha, S.; Babu, R.; Pant, M.C.; et al. Quality protein maize for nutritional security: Rapid development of short duration hybrids through molecular marker assisted breeding. Curr. Sci. 2009, 96, 230-237.

6. Omura, M.; Shimada, T. Citrus breeding, genetics and genomics in Japan. Breed. Sci. 2016, 66, 3-17. [CrossRef]

7. $\mathrm{Xu}, \mathrm{Y}$;; Crouch, J.H. Marker-assisted selection in plant breeding: From publications to practice. Crop Sci. 2008, 48, 391-407. [CrossRef]

8. Yu, J.; Dossa, K.; Wang, L.; Zhang, Y.; Wei, X.; Liao, B.; Zhang, X. PMDBase: A database for studying microsatellite DNA and marker development in plants. Nucleic Acids Res. 2017, 45, D1046-D1053. [CrossRef]

9. Kalia, R.K.; Rai, M.K.; Kalia, S.; Singh, R.; Dhawan, A.K. Microsatellite markers: An overview of the recent progress in plants. Euphytica 2011, 177, 309-334. [CrossRef]

10. Xu, X.; Peng, M.; Fang, Z.; Xu, X. The direction of microsatellite mutations is dependent upon allele length. Nat. Genet. 2000, 24, 396-399. [CrossRef]

11. Wierdl, M.; Dominska, M.; Petes, T.D. Microsatellite instability in yeast: Dependence on the length of the microsatellite. Genetics 1997, 146, 769-779.

12. Akemi, A.; Pereira, J.; Macedo, P.; Alessandra, K. Microsatellites as Tools for Genetic Diversity Analysis. Genet. Divers. Microorg. 2012. [CrossRef]

13. Jiao, Y.; Jia, H.; Li, X.; Chai, M.; Jia, H.; Chen, Z.; Wang, G.; Chai, C.; van de Weg, E.; Gao, Z. Development of simple sequence repeat (SSR) markers from a genome survey of Chinese bayberry (Myrica rubra). BMC Genom. 2012, 13, 201. [CrossRef] [PubMed]

14. Liu, S.-R.; Li, W.-Y.; Long, D.; Hu, C.-G.; Zhang, J.-Z. Development and Characterization of Genomic and Expressed SSRs in Citrus by Genome-Wide Analysis. PLoS ONE 2013, 8, e75149. [CrossRef]

15. Shrestha, R.L.; Dhakal, D.D.; Gautum, D.M.; Paudyal, K.P.; Shrestha, S.; Shrestha, R.L.; Dhakal, D.D.; Gautum, D.M.; Paudyal, K.P.; Shrestha, S. Genetic Diversity Assessment of Acid Lime (Citrus aurantifolia Swingle) Landraces in Nepal, Using SSR Markers. Am. J. Plant Sci. 2012, 3, 1674-1681. [CrossRef]

16. Biswas, M.K.; Xu, Q.; Mayer, C.; Deng, X. Genome Wide Characterization of Short Tandem Repeat Markers in Sweet Orange (Citrus sinensis). PLoS ONE 2014, 9, e104182. [CrossRef] 
17. Ollitrault, F.; Terol, J.; Pina, J.A.; Navarro, L.; Talon, M.; Ollitrault, P. Development of SSR markers from Citrus clementina (Rutaceae) BAC end sequences and interspecific transferability in Citrus. Am. J. Bot. 2010, 97, e124-e129. [CrossRef]

18. Sharafi, A.A.; Abkenar, A.A.; Sharafi, A.; Masaeli, M. Genetic variation assessment of acid lime accessions collected from south of Iran using SSR and ISSR molecular markers. Physiol. Mol. Biol. Plants 2016, 22, 87-95. [CrossRef]

19. Barkley, N.A.; Roose, M.L.; Krueger, R.R.; Federici, C.T. Assessing genetic diversity and population structure in a citrus germplasm collection utilizing simple sequence repeat markers (SSRs). Theor. Appl. Genet. 2006, 112, 1519-1531. [CrossRef]

20. Fanciullino, A.L.; Dhuique-Mayer, C.; Luro, F.; Morillon, R.; Ollitrault, P. Carotenoid biosynthetic pathway in the Citrus genus: Number of copies and phylogenetic diversity of seven genes. J. Agric. Food Chem. 2007, 55, 7405-7417. [CrossRef]

21. Luro, F.L.; Costantino, G.; Terol, J.; Argout, X.; Allario, T.; Wincker, P.; Talon, M.; Ollitrault, P.; Morillon, R. Transferability of the EST-SSRs developed on Nules clementine (Citrus clementina Hort ex Tan) to other citrus species and their effectiveness for genetic mapping. BMC Genom. 2008, 9, 287. [CrossRef]

22. Jiang, D.; Zhong, G.Y.; Hong, Q.B. Analysis of microsatellites in citrus unigenes. Acta Genet. Sin. 2006, 33, 345-353. [CrossRef]

23. Zane, L.; Bargelloni, L.; Patarnello, T. Strategies for microsatellite isolation: A review. Mol. Ecol. 2002, 11, 1-16. [CrossRef] [PubMed]

24. Sharma, P.C.; Grover, A.; Kahl, G. Mining microsatellites in eukaryotic genomes. Trends Biotechnol. 2007, 25, 490-498. [CrossRef] [PubMed]

25. Kwon, Y.S.; Lee, J.M.; Yi, G.B.; Yi, S.I.; Kim, K.M.; Soh, E.H.; Bae, K.M.; Park, E.K.; Song, I.H.; Kim, B.D. Use of SSR markers to complement tests of distinctiveness, uniformity, and stability (DUS) of pepper (Capsicum annuum L.) varieties. Mol. Cells 2005, 19, 428-435. [PubMed]

26. Pourabed, E.; Jazayeri Noushabadi, M.R.; Jamali, S.H.; Moheb Alipour, N.; Zareyan, A.; Sadeghi, L. Identification and DUS testing of rice varieties through microsatellite markers. Int. J. Plant Genom. 2015, 2015, 1-7. [CrossRef] [PubMed]

27. Thirusendura Selvi, D.; Srimathi, P.; Senthil, N.; Ganesan, K.N. Distinctness, uniformity and stability (DUS) characterization on phenological traits and assessing the diversity of inbreds in maize (Zea mays L.). Afr. J. Agric. Res. 2013, 8, 6086-6092. [CrossRef]

28. Vishnu, K. Characterization for DUS descriptors and environmental interaction studies for grain protein and starch content in barley (H. vulgare). Sabrao J. Breed. Genet. Pagination 2015, 260, 267.

29. He, B.; Geng, R.; Cheng, L.; Yang, X.; Ge, H.; Ren, M. Genetic diversity and fingerprinting of 33 standard flue-cured tobacco varieties for use in distinctness, uniformity, and stability testing. BMC Plant Biol. 2020, 20, 378. [CrossRef]

30. Bisen, A.; Khare, D.; Nair, P.; Tripathi, N. SSR analysis of 38 genotypes of soybean (Glycine Max (L.) Merr.) genetic diversity in India. Physiol. Mol. Biol. Plants 2014, 21, 109-115. [CrossRef]

31. Wang, L.X.; Qiu, J.; Chang, L.F.; Liu, L.H.; Li, H.B.; Pang, B.S.; Zhao, C.P. Assessment of wheat variety distinctness using SSR markers. J. Integr. Agric. 2015, 14, 1923-1935. [CrossRef]

32. Molla, R.; Ahmed, I.; Rohman, M.; Hossain, A.; Chowdhury, A.Z. Genetic Diversity Analysis and DNA Fingerprinting of Mungbean (Vigna radiata L.) Genotypes Using SSR Markers. J. Plant Sci. 2016, 4, 153. [CrossRef]

33. Selvan, R.T.; Parthiban, K.T.; Palanikumaran, B. Distinctness, Uniformity and Stability (DUS) Characterization of Neolamarckia Cadamba Genetic Resources. Curr. Agric. Res. J. 2019, 7, 268-275. [CrossRef]

34. Reid, A.; Kerr, E.M. A rapid simple sequence repeat (SSR)-based identification method for potato cultivars. Plant Genet. Resour. 2007, 5, 7-13. [CrossRef]

35. Moore, S.S.; Sargeant, L.L.; King, T.J.; Mattick, J.S.; Georges, M.; Hetzel, D.J. The conservation of dinucleotide microsatellites among mammalian genomes allows the use of heterologous PCR primer pairs in closely related species. Genomics 1991, 10, 654-660. [CrossRef]

36. Gao, L.Z.; Zhang, C.H.; Jia, J.Z. Cross-species Transferability of Rice Microsatellites in its Wild Relatives and the Potential for Conservation Genetic Studies. Genet. Resour. Crop Evol. 2005, 52, 931-940. [CrossRef]

37. Du, L.; Liu, Q.; Zhao, K.; Tang, J.; Zhang, X.; Yue, B.; Fan, Z. PSMD: An extensive database for pan-species microsatellite investigation and marker development. Mol. Ecol. Resour. 2020, 20, 283-291. [CrossRef] 
38. Mokhtar, M.M.; Atia, M.A.M. SSRome: An integrated database and pipelines for exploring microsatellites in all organisms. Nucleic Acids Res. 2019, 47, D244-D252. [CrossRef] [PubMed]

39. Xu, Q.; Chen, L.L.; Ruan, X.; Chen, D.; Zhu, A.; Chen, C.; Bertrand, D.; Jiao, W.B.; Hao, B.H.; Lyon, M.P.; et al. The draft genome of sweet orange (Citrus sinensis). Nat. Genet. 2013, 45, 59-66. [CrossRef] [PubMed]

40. Wu, G.A.; Prochnik, S.; Jenkins, J.; Salse, J.; Hellsten, U.; Murat, F.; Perrier, X.; Ruiz, M.; Scalabrin, S.; Terol, J.; et al. Sequencing of diverse mandarin, pummelo and orange genomes reveals complex history of admixture during citrus domestication. Nat. Biotechnol. 2014, 32, 656-662. [CrossRef]

41. Wang, X.; Xu, Y.; Zhang, S.; Cao, L.; Huang, Y.; Cheng, J.; Wu, G.; Tian, S.; Chen, C.; Liu, Y.; et al. Genomic analyses of primitive, wild and cultivated citrus provide insights into asexual reproduction. Nat. Genet. 2017, 49, 765-772. [CrossRef]

42. Citrus Genome Database. Available online: https://www.citrusgenomedb.org/ (accessed on 13 November 2020).

43. Untergasser, A.; Cutcutache, I.; Koressaar, T.; Ye, J.; Faircloth, B.C.; Remm, M.; Rozen, S.G. Primer3-new capabilities and interfaces. Nucleic Acids Res. 2012, 40, e115. [CrossRef] [PubMed]

44. Morgante, M.; Hanafey, M.; Powell, W. Microsatellites are preferentially associated with nonrepetitive DNA in plant genomes. Nat. Genet. 2002, 30, 194-200. [CrossRef] [PubMed]

45. Katti, M.V.; Ranjekar, P.K.; Gupta, V.S. Differential distribution of simple sequence repeats in eukaryotic genome sequences. Mol. Biol. Evol. 2001, 18, 1161-1167. [CrossRef] [PubMed]

46. Tóth, G.; Gáspári, Z.; Jurka, J. Microsatellites in different eukaryotic genomes: Survey and analysis. Genome Res. 2000, 10, 967-981. [CrossRef]

47. Srivastava, S.; Avvaru, A.K.; Sowpati, D.T.; Mishra, R.K. Patterns of microsatellite distribution across eukaryotic genomes. BMC Genom. 2019, 20, 153. [CrossRef] [PubMed]

48. Zhao, X.; Tian, Y.; Yang, R.; Feng, H.; Ouyang, Q.; Tian, Y.; Tan, Z.; Li, M.; Niu, Y.; Jiang, J.; et al. Coevolution between simple sequence repeats (SSRs) and virus genome size. BMC Genom. 2012, 13, 435. [CrossRef]

49. Portis, E.; Portis, F.; Valente, L.; Moglia, A.; Barchi, L.; Lanteri, S.; Acquadro, A. A genome-wide survey of the microsatellite content of the globe artichoke genome and the development of a web-based database. PLoS ONE 2016, 11, e0162841. [CrossRef]

50. Portis, E.; Lanteri, S.; Barchi, L.; Portis, F.; Valente, L.; Toppino, L.; Rotino, G.L.; Acquadro, A. Comprehensive Characterization of Simple Sequence Repeats in Eggplant (Solanum melongena L.) Genome and Construction of a Web Resource. Front. Plant Sci. 2018, 9, 401. [CrossRef]

51. Haseneyer, G.; Schmutzer, T.; Seidel, M.; Zhou, R.; Mascher, M.; Schön, C.C.; Taudien, S.; Scholz, U.; Stein, N.; Mayer, K.F.X.; et al. From RNA-seq to large-scale genotyping-Genomics resources for rye (Secale cereale L.). BMC Plant Biol. 2011, 11, 131. [CrossRef]

52. Kariin, S.; Burge, C. Dinucleotide relative abundance extremes: A genomic signature. Trends Genet. 1995, 11, 283-290. [CrossRef]

53. Shioiri, C.; Takahata, N. Skew of mononucleotide frequencies, relative abundance of dinucleotides, and DNA strand asymmetry. J. Mol. Evol. 2001, 53, 364-376. [CrossRef] [PubMed]

54. Iquebal, M.A.; Arora, V.; Verma, N.; Rai, A.; Kumar, D. First whole genome based microsatellite DNA marker database of tomato for mapping and variety identification. BMC Plant Biol. 2013, 13, 197. [CrossRef]

55. Zietkiewicz, E.; Rafalski, A.; Labuda, D. Genome fingerprinting by simple sequence repeat (SSR)-anchored polymerase chain reaction amplification. Genomics 1994, 20, 176-183. [CrossRef] [PubMed]

56. Karakousis, A.; Barr, A.R.; Chalmers, K.J.; Ablett, G.A.; Holton, T.A.; Henry, R.J.; Lim, P.; Langridge, P. Potential of SSR markers for plant breeding and variety identification in Australian barley germplasm. Aust. J. Agric. Res. 2003, 54, 1197-1210. [CrossRef]

57. Ali, A.; Jin-Da, W.; Yong-Bao, P.; Zu-Hu, D.; Zhi-Wei, C.; Ru-Kai, C.; San-Ji, G. Molecular Identification and Genetic Diversity Analysis of Chinese Sugarcane (Saccharum spp. Hybrids) Varieties using SSR Markers. Trop. Plant Biol. 2017, 10, 194-203. [CrossRef]

58. Stàgel, A.; Portis, E.; Toppino, L.; Rotino, G.L.; Lanteri, S. Gene-based microsatellite development for mapping and phylogeny studies in eggplant. BMC Genom. 2008, 9, 357. [CrossRef] [PubMed]

59. Shirasawa, K.; Ishii, K.; Kim, C.; Ban, T.; Suzuki, M.; Ito, T.; Muranaka, T.; Kobayashi, M.; Nagata, N.; Isobe, S.; et al. Development of Capsicum EST-SSR markers for species identification and in silico mapping onto the tomato genome sequence. Mol. Breed. 2013, 31, 101-110. [CrossRef]

60. Bhattacharyya, U.; Pandey, S.K.; Dasgupta, T. Identification of EST-SSRs and FDM in sesame (Sesamum indicum L.) through data mining. Sch. J. Agric. Sci. 2014, 4, 60-69. 
61. Goto, S.; Yoshioka, T.; Ohta, S.; Kita, M.; Hamada, H.; Shimizu, T. QTL mapping of male sterility and transmission pattern in progeny of Satsuma mandarin. PLOS ONE 2018, 13, e0200844. [CrossRef]

62. Hong, Q.B.; Ma, X.J.; Gong, G.Z.; Peng, Z.C.; He, Y.R. QTL mapping of citrus freeze tolerance. Acta Hortic. 2015, 1065, 467-474. [CrossRef]

63. Biswas, M.K.; Liu, Y.; Li, C.; Sheng, O.; Mayer, C.; Yi, G. Genome-Wide Computational Analysis of Musa Microsatellites: Classification, Cross-Taxon Transferability, Functional Annotation, Association with Transposons \& miRNAs, and Genetic Marker Potential. PLoS ONE 2015, 10, e0131312. [CrossRef]

Publisher's Note: MDPI stays neutral with regard to jurisdictional claims in published maps and institutional affiliations.

(C) 2020 by the authors. Licensee MDPI, Basel, Switzerland. This article is an open access article distributed under the terms and conditions of the Creative Commons Attribution (CC BY) license (http://creativecommons.org/licenses/by/4.0/). 